\title{
On seismic interferometry, the generalized optical theorem, and the scattering matrix of a point scatterer
}

\author{
Kees Wapenaar ${ }^{1}$, Evert Slob ${ }^{1}$, and Roel Snieder ${ }^{2}$
}

\begin{abstract}
We have analyzed the far-field approximation of the Green's function representation for seismic interferometry. By writing each of the Green's functions involved in the correlation process as a superposition of a direct wave and a scattered wave, the Green's function representation is rewritten as a superposition of four terms. When the scattered waves are modeled with the Born approximation, it appears that a three-term approximation of the Green's function representation (omitting the term containing the crosscorrelation of the scattered waves) yields a nearly exact retrieval, whereas the full four-term expression leads to a significant nonphysical event. This is because the Born approximation does not conserve energy and therefore is an insufficient model to explain all aspects of seismic interferometry. We use the full fourterm expression of the Green's function representation to derive the generalized optical theorem. Unlike other recent derivations, which use stationary phase analysis, our derivation uses reciprocity theory. From the generalized optical theorem, we derive the nonlinear scattering matrix of a point scatterer. This nonlinear model accounts for primary and multiple scattering at the point scatterer and conforms with well-established scattering theory of classical waves. The model is essential to explain fully the results of seismic interferometry, even when it is applied to the response of a single point scatterer. The nonlinear scattering matrix also has implications for modeling, inversion, and migration.
\end{abstract}

\section{INTRODUCTION}

The aim of this paper is to discuss links between the theory of seismic interferometry, the generalized optical theorem, and the scattering matrix of a point scatterer. Seismic interferometry is the method- ology by which new seismic responses are created by crosscorrelating existing responses from controlled transient sources or from random noise sources. For a review, refer to Larose et al. (2006), Schuster (2009), Snieder et al. (2009a), and references therein. The history of the optical theorem starts in the late nineteenth century when Rayleigh and others formulated the relation between the optical refraction index of a scattering medium and its forward scattering amplitude. During World War II, Heisenberg (1943) derived a more general theorem for the scattering matrix (i.e., the angle-dependent scattering amplitude) from quantum mechanics, known as the generalized optical theorem. For a review, refer to Newton (1976) and Marston (2001).

Recently, researchers have recognized that the Green's function representation used in seismic interferometry resembles the generalized optical theorem (Snieder et al., 2008). We show that the generalized optical theorem can be obtained as a special case from the Green's function representation for interferometry. The derivation is similar to that by Snieder et al. (2008) and Halliday and Curtis (2009a) in the sense that we substitute far-field expressions for direct and scattered waves in the Green's function representation. However, instead of using stationary phase analysis, we use reciprocity theory to analyze this representation, term by term. By comparing the final result with the original Green's function representation, the generalized optical theorem follows straightforwardly.

Next, we use the optical theorem to derive the scattering matrix of a point scatterer. We obtain an expansion for which the different terms account for primary and multiple scattering at the point scatterer (van Rossum and Nieuwenhuizen, 1999). We close the circle by analyzing seismic interferometry for the response of a point scatterer. This analysis shows that even for the simple situation of a single point scatterer, the Born approximation does not suffice; the nonlinear scattering matrix is required to completely explain the seismic interferometric result. We conclude with a brief discussion of the implications of the nonlinear scattering aspect of point scatterers for modeling, inversion, and migration.

Presented at the 79th Annual Meeting, SEG. Manuscript received by the Editor 28 July 2009; revised manuscript received 7 November 2009; published online 27 April 2010

${ }^{1}$ Delft University of Technology, Department of Geotechnology, Delft, The Netherlands.E-mail: c.p.a.wapenaar@tudelft.nl; e.c.slob@tudelft.nl.

${ }^{2}$ Colorado School of Mines, Center for Wave Phenomena, Golden, Colorado, U.S.A. E-mail: rsnieder@ mines.edu.

(C) 2010 Society of Exploration Geophysicists. All rights reserved. 


\section{REVIEW OF GREEN'S FUNCTION REPRESENTATION FOR SEISMIC INTERFEROMETRY}

We briefly review the derivation of the Green's function representation for seismic interferometry. Our starting point is the acoustic reciprocity theorem of the correlation type in the space-frequency $(\mathbf{x}, \omega)$ domain for an arbitrary spatial domain $\mathbb{D}$ enclosed by boundary $\partial \mathrm{D}$ with outward-pointing normal vector $\mathbf{n}=\left(n_{1}, n_{2}, n_{3}\right)$ (Morse and Feshbach, 1953; Bojarski, 1983; de Hoop, 1988; Fokkema and van den Berg, 1993):

$$
\oint_{\partial \mathrm{D}} \frac{1}{\rho}\left(\hat{p}_{A}^{*}\left(\partial_{i} \hat{p}_{B}\right)-\left(\partial_{i} \hat{p}_{A}^{*}\right) \hat{p}_{B}\right) n_{i} \mathrm{~d}^{2} \mathbf{x}=\int_{\mathbb{D}}\left\{\hat{s}_{A}^{*} \hat{p}_{B}-\hat{p}_{A}^{*} \hat{s}_{B}\right\} \mathrm{d}^{3} \mathbf{x} .
$$

Here, $\hat{p}(\mathbf{x}, \omega)$ denotes the acoustic pressure and $\hat{s}(\mathbf{x}, \omega)$ is a source distribution; the asterisk denotes complex conjugation. The circumflex above a variable denotes the Fourier transform of the corresponding time-dependent variable, according to $\hat{p}(\mathbf{x}, \omega)=$ $\int \exp (-j \omega t) p(\mathbf{x}, t) \mathrm{d} t(j$ is the imaginary unit). Subscripts $A$ and $B$ distinguish two independent acoustic states in one and the same arbitrary inhomogeneous lossless medium, with mass density $\rho(\mathbf{x})$ and propagation velocity $c(\mathbf{x})$.

If we choose point sources $\hat{s}_{A}(\mathbf{x}, \omega)=\delta\left(\mathbf{x}-\mathbf{x}_{A}\right)$ and $\hat{s}_{B}(\mathbf{x}, \omega)$ $=\delta\left(\mathbf{x}-\mathbf{x}_{B}\right)$, the corresponding acoustic pressure fields in states $A$ and $B$ are Green's functions; hence, $\hat{p}_{A}(\mathbf{x}, \omega)=\hat{G}\left(\mathbf{x}, \mathbf{x}_{A}, \omega\right)$ and $\hat{p}_{B}(\mathbf{x}, \omega)=\hat{G}\left(\mathbf{x}, \mathbf{x}_{B}, \omega\right)$, respectively (see Table 1). Substituting into equation 1 , assuming $\mathbf{x}_{A}$ and $\mathbf{x}_{B}$ are both situated in $\mathbb{D}$, and using source-receiver reciprocity gives

$$
\begin{aligned}
\oint_{\partial \mathrm{D}} \frac{1}{\rho(\mathbf{x})} & \left(\hat{G}^{*}\left(\mathbf{x}, \mathbf{x}_{A}, \omega\right) \partial_{i} \hat{G}\left(\mathbf{x}, \mathbf{x}_{B}, \omega\right)\right. \\
& \left.-\left(\partial_{i} \hat{G}^{*}\left(\mathbf{x}, \mathbf{x}_{A}, \omega\right)\right) \hat{G}\left(\mathbf{x}, \mathbf{x}_{B}, \omega\right)\right) n_{i} \mathrm{~d}^{2} \mathbf{x}=\hat{G}\left(\mathbf{x}_{B}, \mathbf{x}_{A}, \omega\right) \\
& -\hat{G}^{*}\left(\mathbf{x}_{B}, \mathbf{x}_{A}, \omega\right)=2 j \mathfrak{\jmath}\left\{\hat{G}\left(\mathbf{x}_{B}, \mathbf{x}_{A}, \omega\right)\right\},
\end{aligned}
$$

where $\mathfrak{I}$ denotes the imaginary part.

This exact Green's function representation is the basis for seismic interferometry (van Manen et al., 2005; Wapenaar et al., 2005). The right-hand side of equation 2 is the Fourier transform of $G\left(\mathbf{x}_{B}, \mathbf{x}_{A}, t\right)$ $-G\left(\mathbf{x}_{B}, \mathbf{x}_{A},-t\right)$, which is the Green's function of a source at $\mathbf{x}_{A}$ and a receiver at $\mathbf{x}_{B}$, minus its time-reversed version. The products under the integral on the left-hand side correspond to crosscorrelations in the time domain. The left-hand side can be simplified further for a distribution of uncorrelated noise sources on $\partial \mathrm{D}$, but this is beyond the scope of our paper. Note that in previous work we use a slightly differently defined Green's function, leading to a representation of

Table 1. Acoustic states used for the derivation of representation 2.

\begin{tabular}{lcc} 
& State $A$ & State $B$ \\
\hline Wavefields & $\hat{p}_{A}(\mathbf{x}, \omega)=\hat{G}\left(\mathbf{x}, \mathbf{x}_{A}, \omega\right)$ & $\hat{p}_{B}(\mathbf{x}, \omega)=\hat{G}\left(\mathbf{x}, \mathbf{x}_{B}, \omega\right)$ \\
Medium parameters & $\rho(\mathbf{x}), c(\mathbf{x})$ & $\rho(\mathbf{x}), c(\mathbf{x})$ \\
Source functions & $\hat{s}_{A}(\mathbf{x}, \omega)=\delta\left(\mathbf{x}-\mathbf{x}_{A}\right)$ & $\hat{s}_{B}(\mathbf{x}, \omega)=\delta\left(\mathbf{x}-\mathbf{x}_{B}\right)$
\end{tabular}

the real part instead of the imaginary part. Representations for the real or imaginary part are equivalent (Wapenaar and Fokkema, 2006). Here we choose for the form of equation 2 to make the link with the generalized optical theorem more transparent. Equation 2 is also used in the literature on optical holography (Porter, 1970) and inverse scattering (Oristaglio, 1989); see Thorbecke and Wapenaar (2007) for a further discussion.

For the special case of coinciding sources in both states, i.e., for $\mathbf{x}_{A}=\mathbf{x}_{B}=\mathbf{x}_{0}$, equation 2 formulates energy conservation according to

$$
\begin{aligned}
& \oint_{\partial \mathrm{D}} \frac{1}{\rho(\mathbf{x})} \mathfrak{I}\left\{\hat{G}\left(\mathbf{x}, \mathbf{x}_{0}, \omega\right) \partial_{i} \hat{G}^{*}\left(\mathbf{x}, \mathbf{x}_{0}, \omega\right)\right\} n_{i} \mathrm{~d}^{2} \mathbf{x}= \\
& -\mathfrak{I}\left\{\hat{G}\left(\mathbf{x}_{0}, \mathbf{x}_{0}, \omega\right)\right\} .
\end{aligned}
$$

See Snieder et al. (2009b) for a further discussion.

\section{FAR-FIELD APPROXIMATION}

We consider a scattering domain with compact support around the origin, embedded in an otherwise homogeneous medium with mass density $\bar{\rho}$ and propagation velocity $\bar{c}$ (Figure 1 ). We write for the Green's function

$$
\hat{G}\left(\mathbf{x}, \mathbf{x}_{A}, \omega\right)=\hat{\bar{G}}\left(\mathbf{x}, \mathbf{x}_{A}, \omega\right)+\hat{G}^{s}\left(\mathbf{x}, \mathbf{x}_{A}, \omega\right),
$$

where $\hat{\bar{G}}\left(\mathbf{x}, \mathbf{x}_{A}, \omega\right)$ and $\hat{G}^{s}\left(\mathbf{x}, \mathbf{x}_{A}, \omega\right)$ are the direct and scattered wavefields, respectively. The direct wavefield in the embedding is given by

$$
\hat{\bar{G}}\left(\mathbf{x}, \mathbf{x}_{A}, \omega\right)=\frac{\bar{\rho}}{4 \pi} \frac{\exp \left\{-j k\left|\mathbf{x}-\mathbf{x}_{A}\right|\right\}}{\left|\mathbf{x}-\mathbf{x}_{A}\right|},
$$

with $k=\omega / \bar{c}$. Assuming $\mathbf{x}_{A}$ and $\mathbf{x}$ are far from the scattering domain, the scattered wavefield can be written as

$$
\hat{G}^{s}\left(\mathbf{x}, \mathbf{x}_{A}, \omega\right)=\hat{\bar{G}}(\mathbf{x}, \mathbf{0}, \omega) \hat{\theta}\left(\mathbf{k},-\mathbf{k}_{A}\right) \hat{\bar{G}}\left(\mathbf{0}, \mathbf{x}_{A}, \omega\right),
$$

where $\hat{\theta}\left(\mathbf{k},-\mathbf{k}_{A}\right)$ is the complex-valued scattering matrix for an incident wave in the $-\mathbf{k}_{A}$ direction, scattered in the $\mathbf{k}$ direction, with

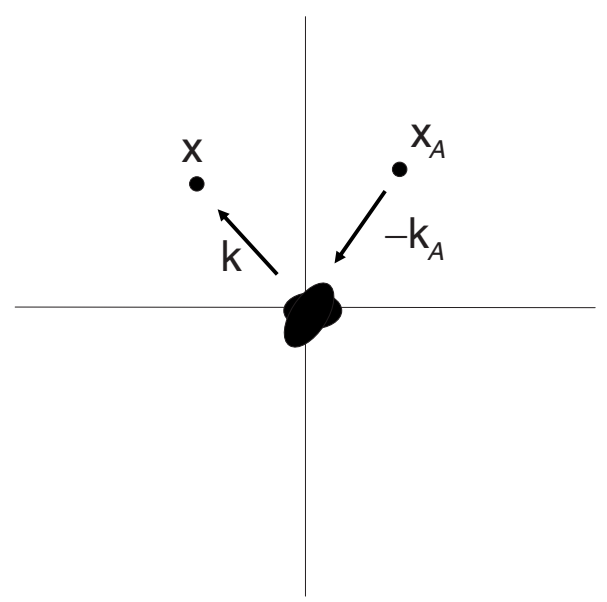

Figure 1. Configuration for far-field expressions - a scattering domain with compact support around the origin, embedded in an otherwise homogeneous medium. 
$\mathbf{k}_{A}=k \mathbf{x}_{A} /\left|\mathbf{x}_{A}\right|$ and $\mathbf{k}=k \mathbf{x} /|\mathbf{x}|$ (Figure 1). Note that the scattering matrix $\hat{\theta}\left(\mathbf{k},-\mathbf{k}_{A}\right)$ accounts for primary and multiple scattering in the scattering domain. Source-receiver reciprocity of the Green's function, i.e., $\hat{G}^{s}\left(\mathbf{x}, \mathbf{x}_{A}, \omega\right)=\hat{G}^{s}\left(\mathbf{x}_{A}, \mathbf{x}, \omega\right)$, implies $\hat{\theta}\left(\mathbf{k},-\mathbf{k}_{A}\right)=$ $\hat{\theta}\left(\mathbf{k}_{A},-\mathbf{k}\right)$.

To facilitate the link with the generalized optical theorem (Glauber and Schomaker, 1953; Newton, 1976), equation 6 can be written alternatively as

$$
\hat{G}^{s}\left(\mathbf{x}, \mathbf{x}_{A}, \omega\right)=\frac{4 \pi}{\bar{\rho}} \hat{\bar{G}}(\mathbf{x}, \mathbf{0}, \omega) f\left(\mathbf{k},-\mathbf{k}_{A}\right) \hat{\bar{G}}\left(\mathbf{0}, \mathbf{x}_{A}, \omega\right),
$$

with $f=(\bar{\rho} / 4 \pi) \hat{\theta}$. The factor $4 \pi / \bar{\rho}$ in equation 7 compensates for $\bar{\rho} / 4 \pi$ in $\hat{\bar{G}}(\mathbf{x}, \mathbf{0}, \omega)$.

Substituting equation 4 and similar expressions for the other Green's functions into the left-hand side of equation 2 gives

$$
\begin{aligned}
\mathcal{L}\left(\hat{G}_{A}, \hat{G}_{B}\right)= & \mathcal{L}\left(\hat{\bar{G}}_{A}, \hat{\bar{G}}_{B}\right)+\mathcal{L}\left(\hat{\bar{G}}_{A}, \hat{G}_{B}^{s}\right)+\mathcal{L}\left(\hat{G}_{A}^{s}, \hat{\bar{G}}_{B}\right) \\
& +\mathcal{L}\left(\hat{G}_{A}^{s}, \hat{G}_{B}^{s}\right)
\end{aligned}
$$

where $\hat{G}_{A}$ stands for $\hat{G}\left(\mathbf{x}, \mathbf{x}_{A}, \omega\right)$, etc., and $\mathcal{L}\left(\hat{G}_{A}, \hat{G}_{B}\right)$ stands for

$$
\begin{aligned}
\mathcal{L}\left(\hat{G}_{A}, \hat{G}_{B}\right)= & \frac{1}{\bar{\rho}} \oint_{\partial \mathrm{D}}\left(\hat{G}^{*}\left(\mathbf{x}, \mathbf{x}_{A}, \omega\right) \partial_{i} \hat{G}\left(\mathbf{x}, \mathbf{x}_{B}, \omega\right)\right. \\
& \left.-\left(\partial_{i} \hat{G}^{*}\left(\mathbf{x}, \mathbf{x}_{A}, \omega\right)\right) \hat{G}\left(\mathbf{x}, \mathbf{x}_{B}, \omega\right)\right) n_{i} \mathrm{~d}^{2} \mathbf{x},
\end{aligned}
$$

etc. In the time domain, these expressions become

$$
\begin{aligned}
\mathcal{L}\left(G_{A}, G_{B}\right)= & \mathcal{L}\left(\bar{G}_{A}, \bar{G}_{B}\right)+\mathcal{L}\left(\bar{G}_{A}, G_{B}^{s}\right)+\mathcal{L}\left(G_{A}^{s}, \bar{G}_{B}\right) \\
& +\mathcal{L}\left(G_{A}^{s}, G_{B}^{s}\right)
\end{aligned}
$$

with

$$
\begin{aligned}
\mathcal{L}\left(G_{A}, G_{B}\right)= & \frac{1}{\bar{\rho}} \oint_{\partial \mathrm{D}}\left(G\left(\mathbf{x}, \mathbf{x}_{A},-t\right) * \partial_{i} G\left(\mathbf{x}, \mathbf{x}_{B}, t\right)\right. \\
& \left.-\partial_{i} G\left(\mathbf{x}, \mathbf{x}_{A},-t\right) * G\left(\mathbf{x}, \mathbf{x}_{B}, t\right)\right) n_{i} \mathrm{~d}^{2} \mathbf{x}
\end{aligned}
$$

etc., where the asterisk denotes temporal convolution.

Before we analyze equation 8 further, we illustrate the different terms in equation 10 with a numerical example.

\section{NUMERICAL EXAMPLE 1: POINT SCATTERER IN BORN APPROXIMATION}

Figure 2 shows the configuration for a 2D numerical experiment; 720 sources are distributed equally along a circle with a radius of $500 \mathrm{~m}$ and its center at the origin. The receiver coordinates are $\mathbf{x}_{A}$ $=(0,-150)$ and $\mathbf{x}_{B}=(200,0)$. The propagation velocity of the homogeneous embedding is $\bar{c}=1500 \mathrm{~m} / \mathrm{s}$, and the mass density $\bar{\rho}$ $=1000 \mathrm{~kg} / \mathrm{m}^{3}$. The star at the origin denotes a point scatterer.

Using source-receiver reciprocity, we write for the Green's functions

$$
\hat{G}\left(\mathbf{x}_{A}, \mathbf{x}, \omega\right)=\hat{\bar{G}}\left(\mathbf{x}_{A}, \mathbf{x}, \omega\right)+\hat{G}^{s}\left(\mathbf{x}_{A}, \mathbf{x}, \omega\right),
$$

with

$$
\hat{G}^{s}\left(\mathbf{x}_{A}, \mathbf{x}, \omega\right)=\hat{\bar{G}}\left(\mathbf{x}_{A}, \mathbf{0}, \omega\right) \hat{\theta}(\omega) \hat{\bar{G}}(\mathbf{0}, \mathbf{x}, \omega)
$$

(and similar expressions for the other Green's functions), where $\hat{\theta}(\omega)$ is the angle-independent scattering matrix. The scatterer is modeled as a contrast in compressibility, according to $\Delta \kappa(\mathbf{x})$ $=\Delta \kappa_{0} \delta(\mathbf{x})$ with $\Delta \kappa_{0}>0$, whereas the density is kept constant. In the Born approximation, the scattering matrix is thus given by $\hat{\theta}(\omega)=\omega^{2} \Delta \kappa_{0}$. In the numerical example, we choose $\Delta \kappa_{0}=6$ $\times 10^{-9} \mathrm{~m}^{3} \mathrm{~Pa}^{-1}$.

The evaluation of the first term in the right-hand side of equation $10, \mathcal{L}\left(\bar{G}_{A}, \bar{G}_{B}\right)$, is illustrated in Figure 3. Figure 3a shows the integrand. Each trace is the result of a crosscorrelation of direct waves $\bar{G}\left(\mathbf{x}_{A}, \mathbf{x}, t\right)$ and $\bar{G}\left(\mathbf{x}_{B}, \mathbf{x}, t\right)$ for one specific source position $\mathbf{x}$ at the surface $\partial \mathrm{D}$. The source coordinate is represented by the angle $\phi$ (conforming with its definition in Figure 2). The Green's functions have been convolved with a Ricker wavelet with a central frequency of $50 \mathrm{~Hz}$. Figure $3 \mathrm{~b}$ shows the result of the integration over the sources. The main contributions come from the Fresnel zones around the stationary points $\mathrm{a}$ and $\mathrm{b}$ in Figures 2 and $3 \mathrm{a}$. The two events in Figure $3 \mathrm{~b}$ correspond to the direct wave $\bar{G}\left(\mathbf{x}_{B}, \mathbf{x}_{A}, t\right)$ and its time-reversed version $-\bar{G}\left(\mathbf{x}_{B}, \mathbf{x}_{A},-t\right)$. The arrival times are $\pm t_{A B}$, with $t_{A B}=\mid \mathbf{x}_{B}$ $-\mathbf{x}_{A} \mid / \bar{c}=0.167 \mathrm{~s}$.

Figure 4 a represents the integrand of $\mathcal{L}\left(\bar{G}_{A}, G_{B}^{s}\right)$, i.e., the second term in the right-hand side of equation 10 . The first stationary point, denoted by c, occurs at $\phi=-90^{\circ}$. For this source, the Green's functions $\bar{G}\left(\mathbf{x}_{A}, \mathbf{x}, t\right)$ and $G^{s}\left(\mathbf{x}_{B}, \mathbf{x}, t\right)$ have the path from $\mathbf{x}$ to $\mathbf{x}_{A}$ in common. Hence, in the crosscorrelation process, the traveltime from $\mathbf{x}$ to $\mathbf{x}_{A}$ is subtracted from that of the scattered Green's function $G^{s}\left(\mathbf{x}_{B}, \mathbf{x}, t\right)$. The remaining traveltime is $t_{A}+t_{B}=\left(\left|\mathbf{x}_{A}\right|+\left|\mathbf{x}_{B}\right|\right) / \bar{c}=0.233 \mathrm{~s}$, the traveltime of the arrival in the scattered Green's function $G^{s}\left(\mathbf{x}_{B}, \mathbf{x}_{A}, t\right)$. Hence, the arrival at $0.233 \mathrm{~s}$ in Figure $4 \mathrm{~b}$ represents $G^{s}\left(\mathbf{x}_{B}, \mathbf{x}_{A}, t\right)$. The second stationary point in Figure $4 \mathrm{a}$, denoted by d, occurs at $\phi=+90^{\circ}$. For this source, the traveltime of the correlation result is $t_{B}-t_{A}=\left(\left|\mathbf{x}_{B}\right|-\left|\mathbf{x}_{A}\right|\right) / \bar{c}=0.033 \mathrm{~s}$. The arrival at $0.033 \mathrm{~s}$ in Figure $4 \mathrm{~b}$ has no physical meaning.

Figure 5a represents the integrand of the third term in the righthand side of equation $10, \mathcal{L}\left(G_{A}^{s}, \bar{G}_{B}\right)$. At the stationary point, denoted by e, the Green's functions $G^{s}\left(\mathbf{x}_{A}, \mathbf{x}, t\right)$ and $\bar{G}\left(\mathbf{x}_{B}, \mathbf{x}, t\right)$ have the path

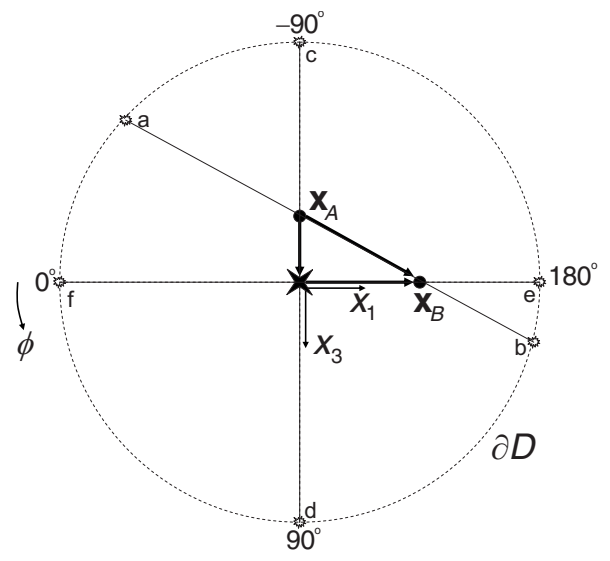

Figure 2. Single point scatterer in a homogeneous embedding. The receivers are at $\mathbf{x}_{A}$ and $\mathbf{x}_{B}$. The numerical integration is carried out along the sources at surface $\partial \mathrm{D}$. The main contributions come from stationary points a, b, c, and e. 
from $\mathbf{x}$ to $\mathbf{x}_{B}$ in common. The traveltime of the correlation result is $-\left(t_{A}+t_{B}\right)=-0.233 \mathrm{~s}$. The arrival at this traveltime in Figure $5 \mathrm{~b}$ represents the time-reversed scattered Green's function $-G^{s}\left(\mathbf{x}_{B}, \mathbf{x}_{A}\right.$, $-t$ ). The stationary point denoted by $\mathrm{f}$ contributes to the nonphysical arrival at $0.033 \mathrm{~s}$ in Figure 5b. Note that this arrival is opposite in sign compared with the arrival at $0.033 \mathrm{~s}$ in Figure 4b.

We now superpose the results of $\mathcal{L}\left(\bar{G}_{A}, \bar{G}_{B}\right), \mathcal{L}\left(\bar{G}_{A}, G_{B}^{s}\right)$, and $\mathcal{L}\left(G_{A}^{s}, \bar{G}_{B}\right)$. Figure 6 shows the sum of the results in Figures 3-5. In this and subsequent displays, the amplitudes of $\mathcal{L}\left(\bar{G}_{A}, \bar{G}_{B}\right)$ $=\bar{G}\left(\mathbf{x}_{B}, \mathbf{x}_{A}, t\right)-\bar{G}\left(\mathbf{x}_{B}, \mathbf{x}_{A},-t\right)$ (Figure 3$)$ are divided by a factor of 20 to avoid clipping. The events in Figure $6 \mathrm{~b}$ are $\bar{G}\left(\mathbf{x}_{B}, \mathbf{x}_{A}, t\right)-\bar{G}\left(\mathbf{x}_{B}, \mathbf{x}_{A}\right.$, $-t)+G^{s}\left(\mathbf{x}_{B}, \mathbf{x}_{A}, t\right)-G^{s}\left(\mathbf{x}_{B}, \mathbf{x}_{A},-t\right)$. Note that the nonphysical arrivals at $0.033 \mathrm{~s}$ cancel each other. Hence, the result in Figure $6 \mathrm{~b}$ represents the complete Green's function between $\mathbf{x}_{A}$ and $\mathbf{x}_{B}$, minus its time-reversed version, i.e., $G\left(\mathbf{x}_{B}, \mathbf{x}_{A}, t\right)-G\left(\mathbf{x}_{B}, \mathbf{x}_{A},-t\right)$. Figure 7 shows the result of Figure $6 \mathrm{~b}$, together with the directly modeled Green's function between $\mathbf{x}_{A}$ and $\mathbf{x}_{B}$. The match is nearly perfect.

Finally, we evaluate the last term on the right-hand side of equation $10, \mathcal{L}\left(G_{A}^{s}, G_{B}^{s}\right)$. The scattered Green's functions $G^{s}\left(\mathbf{x}_{A}, \mathbf{x}, t\right)$ and $G^{s}\left(\mathbf{x}_{B}, \mathbf{x}, t\right)$ have the path from $\mathbf{x}$ to the point scatterer in common for all $\mathbf{x}$. Hence, the traveltime of the correlation result is equal to $t_{B}$ $-t_{A}=\left(\left|\mathbf{x}_{B}\right|-\left|\mathbf{x}_{A}\right|\right) / \bar{c}=0.033 \mathrm{~s}$ for all $\mathbf{x}$ (Figure 8a). The integration result is shown in Figure 8b. Following equation 10, we add this to the other three terms and compare it again with the modeled Green's function (Figure 9). Note the artifact at $0.033 \mathrm{~s}$; the arrival time of this event has no physical meaning. The amplitude is proportional to the energy scattered by the point scatterer.
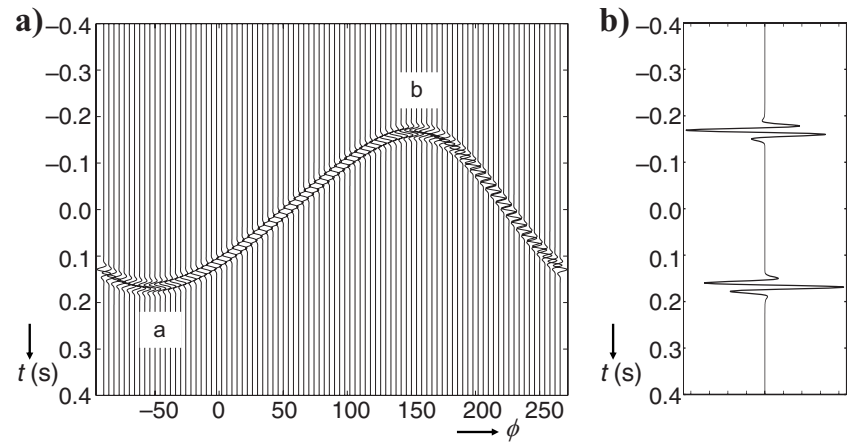

Figure 3. (a) The integrand of $\mathcal{L}\left(\bar{G}_{A}, \bar{G}_{B}\right)$. (b) The sum of all traces in (a). These events represent the direct wave $\bar{G}\left(\mathbf{x}_{B}, \mathbf{x}_{A}, t\right)$ and its timereversed version $-\bar{G}\left(\mathbf{x}_{B}, \mathbf{x}_{A},-t\right)$.
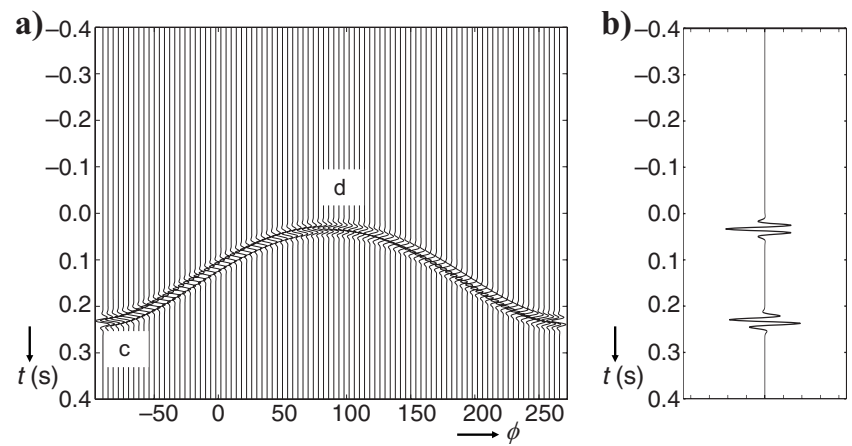

Figure 4. (a) The integrand of $\mathcal{L}\left(\bar{G}_{A}, G_{B}^{s}\right)$. (b) The sum of all traces in (a). The event at $0.233 \mathrm{~s}$ represents the scattered Green's function $G^{s}\left(\mathbf{x}_{B}, \mathbf{x}_{A}, t\right)$; the event at $0.033 \mathrm{~s}$ has no physical meaning.

\section{BORN APPROXIMATION AND BEYOND}

From the numerical example, it appears that taking only three of the four terms of equation 10 into account leads to a better retrieval of the Green's function (Figure 7) than when all terms are taken into account, as prescribed by the theory (Figure 9). In most practical situations, it is not possible to apply the crosscorrelation term by term; when the full responses are crosscorrelated, the fourth term is included automatically. Apart from this practical issue, a more intriguing question is how it is possible that the three-term approximation leads to a better result than the full four-term expression.

The answer is that we use the Born approximation to model the point scatterer. The Born approximation does not conserve energy (Born and Wolf, 1965; Rodberg and Thaler, 1967; Butkov, 1968), so equation 3 and the more general equation 2 are violated. The Born approximation involves only first-order scattering; so to apply equation 2 consistently, we should only consider terms up to first-order scattering (Wapenaar et al., 2005; Halliday and Curtis, 2009b). The first three terms on the right-hand side of equation 10 obey this condition, but the fourth term, $\mathcal{L}\left(G_{A}^{s}, G_{B}^{s}\right)$, describes the crosscorrelation of two scattered Green's functions. Therefore, this term is proportional to second-order scattering and should be omitted to remain consistent with the Born approximation. Apparently, we should go beyond first-order Born modeling if we want a consistent result
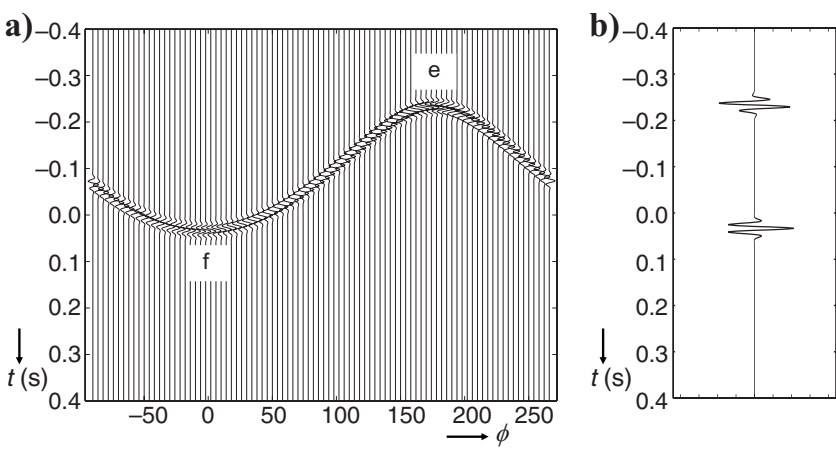

Figure 5. (a) The integrand of $\mathcal{L}\left(G_{A}^{s}, \bar{G}_{B}\right)$. (b) The sum of all traces in (a). The event at $-0.233 \mathrm{~s}$ represents the time-reversed scattered Green's function $-G^{s}\left(\mathbf{x}_{B}, \mathbf{x}_{A},-t\right)$; the event at $0.033 \mathrm{~s}$ has no physical meaning.

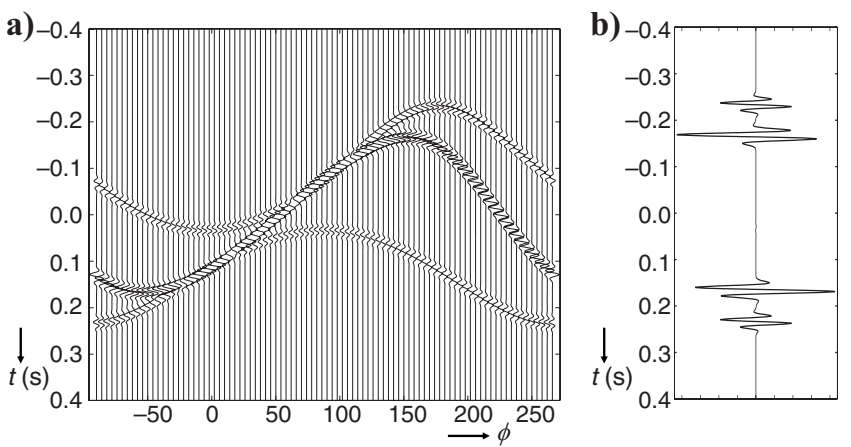

Figure 6. (a) Superposition of Figures 3a, 4a, and 5a. (b) The sum of all traces in (a). This represents the complete Green's function between $\mathbf{x}_{A}$ and $\mathbf{x}_{B}$, minus its time-reversed version, i.e., $G\left(\mathbf{x}_{B}, \mathbf{x}_{A}, t\right)$ $-G\left(\mathbf{x}_{B}, \mathbf{x}_{A},-t\right)$. In this and subsequent displays, the amplitudes of the direct waves, $\bar{G}\left(\mathbf{x}_{B}, \mathbf{x}_{A}, t\right)-\bar{G}\left(\mathbf{x}_{B}, \mathbf{x}_{A},-t\right)$, are divided by 20 to avoid clipping. 
when all four terms of equation 8 or 10 are taken into account. Although they do not mention this aspect explicitly, van Manen et al. (2006) recognize this and use a nonlinear point-scatterer model in their interferometric modeling method.

Snieder et al. (2008) analyze the four terms in the right-hand side of equation 8 by the method of stationary phase for a situation of an arbitrary scattering domain with compact support around the origin. They show that the nonphysical events resulting from the last three terms cancel each other on account of the generalized optical theorem (Glauber and Schomaker, 1953) and thus confirm the consistency of equation 2. Halliday and Curtis (2009a) turn the argument around: Because the Green's function representation 2 is correct, the nonphysical events on the right-hand side of equation 8 must cancel each other and, hence, the generalized optical theorem follows from equation 2. They use this approach to derive a generalized optical theorem for surface waves in layered elastic media.

In the next section, we follow the second route, except that instead of using the method of stationary phase to analyze the integrals, we use the correlation reciprocity theorem (equation 1) to evaluate the different terms on the right-hand side of equation 8 . This approach is more straightforward but does not circumvent the approximations inherent to the stationary phase method.

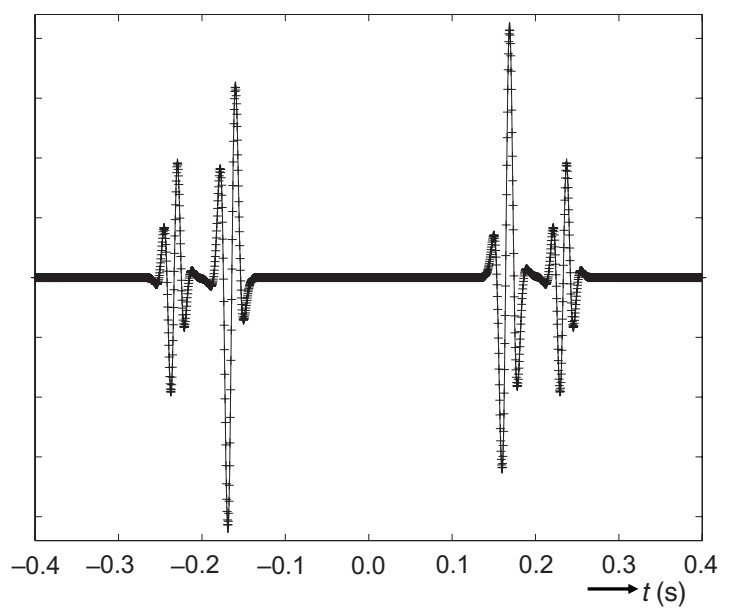

Figure 7. Three-term approximation of equation 10 (i.e., the result of Figure $6 \mathrm{~b}$, here denoted by the solid line), compared with the directly modeled Green's function between $\mathbf{x}_{A}$ and $\mathbf{x}_{B}$ (denoted by the plus signs).
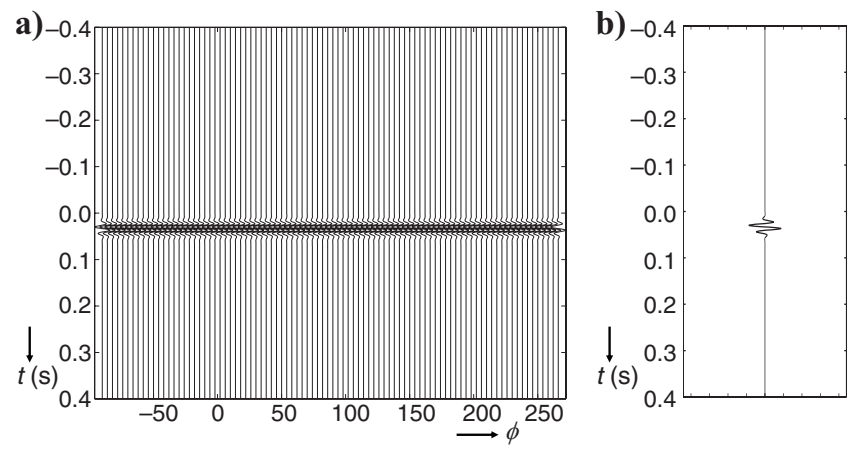

Figure 8. (a) The integrand of $\mathcal{L}\left(G_{A}^{s}, G_{B}^{s}\right)$. (b) The sum of all traces in (a).

\section{DERIVATION OF GENERALIZED OPTICAL THEOREM FROM GREEN'S FUNCTION REPRESENTATION}

We derive the generalized optical theorem from Green's function representation 2 for the configuration of Figure 1, with $\hat{G}\left(\mathbf{x}, \mathbf{x}_{A}, \omega\right)$ defined by equations 4,5 , and 7 . For the boundary $\partial \mathrm{D}$, we choose a sphere with its center at the origin, encompassing $\mathbf{x}_{A}$ and $\mathbf{x}_{B}$. We analyze the four terms on the right-hand side of equation 8 , constituting the integral in equation 2.

The first term on the right-hand side of equation 8 has the same form as $\mathcal{L}\left(\hat{G}_{A}, \hat{G}_{B}\right)$ but with the full Green's functions replaced by Green's functions in the homogeneous embedding. These Green's functions and the medium parameters of the embedding are summarized in Table 2. Substitution into equation 1 gives, analogous to equation 2 ,

$$
\begin{aligned}
\mathcal{L}\left(\hat{\bar{G}}_{A}, \hat{\bar{G}}_{B}\right) & =\hat{\bar{G}}\left(\mathbf{x}_{B}, \mathbf{x}_{A}, \omega\right)-\hat{\bar{G}}^{*}\left(\mathbf{x}_{B}, \mathbf{x}_{A}, \omega\right) \\
& =2 j \mathfrak{\jmath}\left\{\hat{\bar{G}}\left(\mathbf{x}_{B}, \mathbf{x}_{A}, \omega\right)\right\} .
\end{aligned}
$$

For the analysis of the second term on the right-hand side of equation 8, i.e., $\mathcal{L}\left(\hat{\bar{G}}_{A}, \hat{G}_{B}^{s}\right)$, we again use equation 1 .

First, observe that $\hat{\bar{G}}_{A}$ is the response of a point source at $\mathbf{x}_{A}$ in the homogeneous embedding. Hence, for $\hat{s}_{A}$ and $\hat{p}_{A}$, we have $\hat{s}_{A}(\mathbf{x}, \omega)$ $=\delta\left(\mathbf{x}-\mathbf{x}_{A}\right)$ and $\hat{p}_{A}(\mathbf{x}, \omega)=\hat{\bar{G}}\left(\mathbf{x}, \mathbf{x}_{A}, \omega\right)$, respectively (see Table 3, state $A)$. Note that $\hat{p}_{B}(\mathbf{x}, \omega)=\hat{G}^{s}\left(\mathbf{x}, \mathbf{x}_{B}, \omega\right)$ is the scattered response of the actual medium. However, we can only use equation 1 when $\hat{p}_{A}$

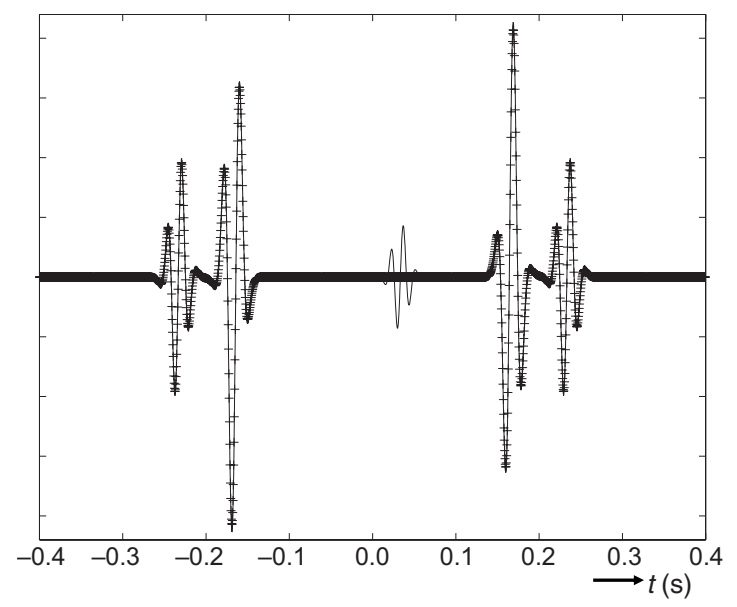

Figure 9. All four terms of equation 10 (i.e., the result of Figure 7, with the event of Figure $8 \mathrm{~b}$ added to it), compared with the directly modeled Green's function between $\mathbf{x}_{A}$ and $\mathbf{x}_{B}$ (denoted by the plus signs).

Table 2. Acoustic states used for the evaluation of $\mathcal{L}\left(\hat{\bar{G}}_{A}, \hat{\bar{G}}_{B}\right)$.

\begin{tabular}{lcc}
\hline & State $A$ & State $B$ \\
\hline Wavefields & $\hat{p}_{A}(\mathbf{x}, \omega)=\hat{\bar{G}}\left(\mathbf{x}, \mathbf{x}_{A}, \omega\right)$ & $\hat{p}_{B}(\mathbf{x}, \omega)=\hat{\bar{G}}\left(\mathbf{x}, \mathbf{x}_{B}, \omega\right)$ \\
Medium parameters & $\bar{\rho}, \bar{c}$ & $\bar{\rho}, \bar{c}$ \\
Source functions & $\hat{s}_{A}(\mathbf{x}, \omega)=\delta\left(\mathbf{x}-\mathbf{x}_{A}\right)$ & $\hat{s}_{B}(\mathbf{x}, \omega)=\delta\left(\mathbf{x}-\mathbf{x}_{B}\right)$ \\
\hline
\end{tabular}


and $\hat{p}_{B}$ are defined in the same medium. Therefore, we rewrite $\hat{p}_{B}$ as the response of an equivalent source distribution in the homogeneous embedding. Thus, $\hat{p}_{B}(\mathbf{x}, \omega)=\hat{\bar{G}}(\mathbf{x}, \mathbf{0}, \omega) \breve{s}_{B}(\mathbf{k}, \omega)$ where, according to equation $7, \breve{s}_{B}(\mathbf{k}, \omega)=(4 \pi / \bar{\rho}) f\left(\mathbf{k},-\mathbf{k}_{B}\right) \hat{\bar{G}}\left(\mathbf{0}, \mathbf{x}_{B}, \omega\right)$. Note that $\hat{\bar{G}}(\mathbf{x}, \mathbf{0}, \omega) \breve{s}_{B}(\mathbf{k}, \omega)$ can be interpreted as the far-field response in the homogeneous embedding of a source distribution $\hat{s}_{B}(\mathbf{x}, \omega)$ with compact support around the origin, assuming that $\breve{s}_{B}(\mathbf{k}, \omega)$ is the $3 \mathrm{D}$ spatial Fourier transform of $\hat{s}_{B}(\mathbf{x}, \omega)$ (Fraunhofer approximation). Hence, the equivalent source distribution $\hat{s}_{B}(\mathbf{x}, \omega)$ generating $\hat{p}_{B}(\mathbf{x}, \omega)$ in the homogeneous embedding is defined as the inverse Fourier transform of $\breve{s}_{B}(\mathbf{k}, \omega)$, i.e., $\hat{s}_{B}(\mathbf{x}, \omega)=(4 \pi / \bar{\rho}) \hat{\bar{G}}\left(\mathbf{0}, \mathbf{x}_{B}, \omega\right)$ $\times(1 / 2 \pi)^{3} \int \exp (-j \mathbf{k} \cdot \mathbf{x}) f\left(\mathbf{k},-\mathbf{k}_{B}\right) \mathrm{d}^{3} \mathbf{k}$ (see Table 3 , state $\left.B\right)$.

We have now defined all of the terms appearing on the right-hand side of equation 1 . Because $\hat{s}_{B}(\mathbf{x}, \omega)$ has compact support, the product $\hat{p}_{A}^{*} \hat{s}_{B}$ is evaluated for small $\mathbf{x}$ only. This justifies the approximation $\hat{p}_{A}(\mathbf{x}, \omega)=\hat{\bar{G}}\left(\mathbf{x}, \mathbf{x}_{A}, \omega\right)=\exp \left(j \mathbf{k}_{A} \cdot \mathbf{x}\right) \hat{\bar{G}}\left(\mathbf{0}, \mathbf{x}_{A}, \omega\right)$ (see Table 3, state $A$ ). Making the appropriate substitutions on the right-hand side of equation 1 , interchanging the integrals over $\mathbf{k}$ and $\mathbf{x}$, using $\int \exp \left(-j\left(\mathbf{k}+\mathbf{k}_{A}\right) \cdot \mathbf{x}\right) \mathrm{d}^{3} \mathbf{x}=(2 \pi)^{3} \delta\left(\mathbf{k}+\mathbf{k}_{A}\right)$ and the sifting-property of the delta function, gives

$$
\begin{aligned}
\mathcal{L}\left(\hat{\bar{G}}_{A}, \hat{G}_{B}^{s}\right)= & \hat{G}^{s}\left(\mathbf{x}_{B}, \mathbf{x}_{A}, \omega\right) \\
& -\frac{4 \pi}{\bar{\rho}} \hat{\bar{G}}^{*}\left(\mathbf{0}, \mathbf{x}_{A}, \omega\right) \hat{\bar{G}}\left(\mathbf{0}, \mathbf{x}_{B}, \omega\right) f\left(-\mathbf{k}_{A},-\mathbf{k}_{B}\right)
\end{aligned}
$$

In a similar way, we find the third term on the right-hand side of equation 8 :

$$
\begin{aligned}
\mathcal{L}\left(\hat{G}_{A}^{s}, \hat{\bar{G}}_{B}\right)= & -\hat{G}^{s, *}\left(\mathbf{x}_{B}, \mathbf{x}_{A}, \omega\right) \\
& +\frac{4 \pi}{\bar{\rho}} \hat{\bar{G}}^{*}\left(\mathbf{0}, \mathbf{x}_{A}, \omega\right) \hat{\bar{G}}\left(\mathbf{0}, \mathbf{x}_{B}, \omega\right) f^{*}\left(-\mathbf{k}_{B},-\mathbf{k}_{A}\right)
\end{aligned}
$$

For the analysis of the fourth term in the right-hand side of equation 8 , we substitute equation 7 and a similar expression for $\hat{G}^{s}\left(\mathbf{x}, \mathbf{x}_{B}, \omega\right)$ into the right-hand side of equation 9. Using $n_{i} \partial_{i} \hat{G}(\mathbf{x}, \mathbf{0}, \omega)=-j k \hat{G}(\mathbf{x}, \mathbf{0}, \omega)$ and $(4 \pi / \bar{\rho})^{2}|\hat{\bar{G}}(\mathbf{x}, \mathbf{0}, \omega)|^{2} \mathrm{~d}^{2} \mathbf{x}=\mathrm{d}^{2} \mathbf{x} /$ $|\mathbf{x}|^{2}=\mathrm{d} \Omega$, this gives

$$
\begin{aligned}
\mathcal{L}\left(\hat{G}_{A}^{s}, \hat{G}_{B}^{s}\right)= & -\frac{2 j k}{\bar{\rho}} \hat{\bar{G}}^{*}\left(\mathbf{0}, \mathbf{x}_{A}, \omega\right) \hat{\bar{G}}\left(\mathbf{0}, \mathbf{x}_{B}, \omega\right) \\
& \times \oint f^{*}\left(\mathbf{k},-\mathbf{k}_{A}\right) f\left(\mathbf{k},-\mathbf{k}_{B}\right) \mathrm{d} \Omega
\end{aligned}
$$

Substituting equations $14-17$ into the right-hand side of equation 8 gives

$$
\begin{aligned}
\oint_{\partial \mathrm{D}} & \frac{1}{\bar{\rho}(\mathbf{x})}\left(\hat{G}^{*}\left(\mathbf{x}, \mathbf{x}_{A}, \omega\right) \partial_{i} \hat{G}\left(\mathbf{x}, \mathbf{x}_{B}, \omega\right)\right. \\
& \left.-\left(\partial_{i} \hat{G}^{*}\left(\mathbf{x}, \mathbf{x}_{A}, \omega\right)\right) \hat{G}\left(\mathbf{x}, \mathbf{x}_{B}, \omega\right)\right) n_{i} \mathrm{~d}^{2} \mathbf{x} \\
= & 2 j \Im\left\{\hat{I}\left(\mathbf{x}_{B}, \mathbf{x}_{A}, \omega\right)\right\}-\frac{8 \pi j}{\bar{\rho}} \hat{\bar{G}}^{*}\left(\mathbf{0}, \mathbf{x}_{A}, \omega\right) \hat{\bar{G}}\left(\mathbf{0}, \mathbf{x}_{B}, \omega\right) \\
& \times\left(\frac{1}{2 j}\left\{f\left(-\mathbf{k}_{A},-\mathbf{k}_{B}\right)-f^{*}\left(-\mathbf{k}_{B},-\mathbf{k}_{A}\right)\right\}\right. \\
& \left.+\frac{k}{4 \pi} \oint f^{*}\left(\mathbf{k},-\mathbf{k}_{A}\right) f\left(\mathbf{k},-\mathbf{k}_{B}\right) \mathrm{d} \Omega\right) .
\end{aligned}
$$

In comparison with equation 2 , the right-hand side contains an extra term that must equal zero (or equation 2 is violated). Hence,

$$
\frac{-1}{2 j}\left\{f\left(\mathbf{k}_{A}, \mathbf{k}_{B}\right)-f^{*}\left(\mathbf{k}_{B}, \mathbf{k}_{A}\right)\right\}=\frac{k}{4 \pi} \oint f^{*}\left(\mathbf{k}, \mathbf{k}_{A}\right) f\left(\mathbf{k}, \mathbf{k}_{B}\right) \mathrm{d} \Omega .
$$

Note that we have renamed $-\mathbf{k}_{A}$ and $-\mathbf{k}_{B}$ as $\mathbf{k}_{A}$ and $\mathbf{k}_{B}$, respectively.

Equation 19 is known as the generalized optical theorem (Heisenberg, 1943; Glauber and Schomaker, 1953; Newton, 1976; Marston, 2001). The first minus sign on the left-hand side is usually absent; it stems from our definition of the temporal Fourier transform. The optical theorem follows by taking $\mathbf{k}_{A}=\mathbf{k}_{B}=\mathbf{k}_{0}$; hence,

$$
-\Im\left\{f\left(\mathbf{k}_{0}, \mathbf{k}_{0}\right)\right\}=\frac{k}{4 \pi} \oint\left|f\left(\mathbf{k}, \mathbf{k}_{0}\right)\right|^{2} \mathrm{~d} \Omega .
$$

For an isotropic scatterer, this reduces to

$$
-\Im(f)=k|f|^{2}
$$

with $f=f(\omega)$.

Table 3. Acoustic states used for evaluating $\mathcal{L}\left(\hat{\bar{G}}_{A}, \hat{G}_{B}^{s}\right)$.

State $A$

State $B$

\section{Wavefields}

Medium parameters

Source functions

$$
\begin{gathered}
\hat{p}_{A}(\mathbf{x}, \omega)=\hat{\bar{G}}\left(\mathbf{x}, \mathbf{x}_{A}, \omega\right)=\exp \left(j \mathbf{k}_{A} \cdot \mathbf{x}\right) \hat{\bar{G}}\left(\mathbf{0}, \mathbf{x}_{A}, \omega\right) \\
\bar{\rho}, \bar{c} \\
\hat{s}_{A}(\mathbf{x}, \omega)=\delta\left(\mathbf{x}-\mathbf{x}_{A}\right)
\end{gathered}
$$

$$
\begin{gathered}
\hat{p}_{B}(\mathbf{x}, \omega)=\hat{G}^{s}\left(\mathbf{x}, \mathbf{x}_{B}, \omega\right)=\hat{\bar{G}}(\mathbf{x}, \mathbf{0}, \omega) \breve{s_{B}}(\mathbf{k}, \omega) \\
\bar{\rho}, \bar{c} \\
\hat{s}_{B}(\mathbf{x}, \omega)=\left(\frac{1}{2 \pi}\right)^{3} \int \exp (-j \mathbf{k} \cdot \mathbf{x}) \breve{s_{B}}(\mathbf{k}, \omega) \mathrm{d}^{3} \mathbf{k}, \\
\breve{s}_{B}(\mathbf{k}, \omega)=\frac{4 \pi}{\bar{\rho}} f\left(\mathbf{k},-\mathbf{k}_{B}\right) \hat{\bar{G}}\left(\mathbf{0}, \mathbf{x}_{B}, \omega\right)
\end{gathered}
$$




\section{DERIVATION OF SCATTERING MATRIX OF A POINT SCATTERER FROM OPTICAL THEOREM}

We use the optical theorem to derive an explicit expression for the full scattering matrix of a nonlinear point scatterer. Here, "nonlinear" refers to the fact that the scattered field is nonlinear in terms of the parameter contrast (but it is linear in terms of the incident wavefield). The field scattered by a point scatterer at $\mathbf{x}=\mathbf{0}$ is given by

$$
\hat{G}^{s}\left(\mathbf{x}, \mathbf{x}_{A}, \omega\right)=\hat{\bar{G}}(\mathbf{x}, \mathbf{0}, \omega) \hat{\theta}(\omega) \hat{\bar{G}}\left(\mathbf{0}, \mathbf{x}_{A}, \omega\right),
$$

with $\hat{\theta}(\omega)=(4 \pi / \bar{\rho}) f(\omega)$. We rewrite the optical theorem for an isotropic scatterer (equation 21) as

$$
-\Im(\hat{\theta})=\frac{k \bar{\rho}}{4 \pi}|\hat{\theta}|^{2} .
$$

For a compressibility contrast, we had for the first-order Born approximation $\hat{\theta}_{1}=\omega^{2} \Delta \kappa_{0}$, with $\Delta \kappa_{0}>0$. We use equation 23 to derive the imaginary part of the second-order Born approximation $\hat{\theta}_{2}$ :

$$
\hat{\theta}_{2}(\omega)=\hat{\theta}_{1}(\omega)-j \frac{k \bar{\rho}}{4 \pi} \hat{\theta}_{1}^{2}(\omega) .
$$

Compare this with the following expression of van Rossum and Nieuwenhuizen (1999)

$$
\hat{\theta}_{2}(\omega)=\hat{\theta}_{1}(\omega)+\hat{\theta}_{1}(\omega) \hat{\bar{G}}^{\mathrm{reg}}(\mathbf{0 , 0}, \omega) \hat{\theta}_{1}(\omega)
$$

where $\hat{\bar{G}}^{\text {reg }}(\mathbf{0}, \mathbf{0}, \omega)$ is a regularized version of the return Green's function $\hat{\bar{G}}(\mathbf{0}, \mathbf{0}, \omega)$. The real part of $\hat{\bar{G}}(\mathbf{0 , 0}, \omega)$ diverges, but van Rossum and Nieuwenhuizen (1999) argue that "in reality these divergences are cut off by the physical size of the scatterer, so they play no role for weak scattering." Therefore, they only keep the imaginary part; thus (using equation 5),

$$
\begin{aligned}
\hat{\bar{G}}^{\mathrm{reg}}(\mathbf{0}, \mathbf{0}, \omega) & =\lim _{|\mathbf{x}| \rightarrow 0} j \Im\{\hat{\bar{G}}(\mathbf{x}, \mathbf{0}, \omega)\}=-j \frac{\bar{\rho}}{4 \pi} \lim _{|\mathbf{x}| \rightarrow 0} \frac{\sin (k|\mathbf{x}|)}{|\mathbf{x}|} \\
& =-j \frac{k \bar{\rho}}{4 \pi} .
\end{aligned}
$$

With this expression for the regularized Green's function, our second-order Born approximation derived from the optical theorem (equation 24) is identical with equation 25. Analogous to an expression for nonlinear 1D scattering derived by Snieder (1999), we account for higher-order scattering at the point scatterer as

$$
\hat{\theta}(\omega)=\hat{\theta}_{1}+\hat{\theta}_{1} \hat{\bar{G}}_{0}^{\mathrm{reg}} \hat{\theta}_{1}+\hat{\theta}_{1} \hat{\bar{G}}_{0}^{\mathrm{reg}} \hat{\theta}_{1} \hat{\bar{G}}_{0}^{\mathrm{reg}} \hat{\theta}_{1}+\cdots
$$

where $\hat{\bar{G}}_{0}^{\text {reg }}$ stands for $\hat{\bar{G}}^{\text {reg }}(\mathbf{0 , 0}, \omega)$. This expansion for primary and multiple scattering is illustrated in Figure 10. Each term corresponds to a causal operation in the time domain, so the expansion is causal as well.

Equation 27 converges for $\left|\hat{\theta}_{1} \hat{\bar{G}}_{0}^{\text {reg }}\right|=\left(\bar{\rho} \Delta \kappa_{0} / 4 \pi \bar{c}\right) \omega^{3}<1$, i.e., for small contrasts and finite frequencies. It can be written in closed form as

$$
\hat{\theta}=\frac{\hat{\theta}_{1}}{1-\hat{\theta}_{1} \hat{\bar{G}}_{0}^{\mathrm{reg}}} \text { for }\left|\hat{\theta}_{1} \hat{\bar{G}}_{0}^{\mathrm{reg}}\right|=\frac{\bar{\rho} \Delta \kappa_{0}}{4 \pi \bar{c}} \omega^{3}<1
$$

This expression for the scattering matrix $\hat{\theta}$, with $\hat{\bar{G}}_{0}^{\text {reg }}$ defined by equation 26, obeys the optical theorem (equation 23). Van Rossum and Nieuwenhuizen (1999) discuss equation 28 further for the situation when $\hat{\bar{G}}_{0}^{\text {reg }}$ is the regularized return Green's function for a scatterer with finite size.

\section{NUMERICAL EXAMPLE 2: NONLINEAR POINT SCATTERER}

We repeat the numerical experiments, this time using a nonlinear scatterer model. Because we consider a 2D configuration, equations 23 and 28 need to be replaced by their $2 \mathrm{D}$ counterparts

$$
-\Im(\hat{\theta})=\operatorname{sgn}(\omega) \frac{\bar{\rho}}{4}|\hat{\theta}|^{2}
$$

and

$$
\hat{\theta}=\frac{\hat{\theta}_{1}}{1+j \hat{\theta}_{1} \operatorname{sgn}(\omega) \frac{\bar{\rho}}{4}} \text { for } \frac{\bar{\rho} \Delta \kappa_{0}}{4} \omega^{2}<1,
$$

respectively, with $\hat{\theta}_{1}(\omega)=\omega^{2} \Delta \kappa_{0}$. Figure 11 shows the result of the first three terms of equation 10, i.e., $\mathcal{L}\left(\bar{G}_{A}, \bar{G}_{B}\right)+\mathcal{L}\left(\bar{G}_{A}, G_{B}^{s}\right)$ $+\mathcal{L}\left(G_{A}^{s}, \bar{G}_{B}\right)$. Note that the nonphysical arrivals at $t_{B}-t_{A}=0.033 \mathrm{~s}$ resulting from $\mathcal{L}\left(\bar{G}_{A}, G_{B}^{s}\right)$ and $\mathcal{L}\left(G_{A}^{s}, \bar{G}_{B}\right)$ do not cancel. Unlike in Figures 4 and 5, where these events showed a zero-phase behavior, here they are not zero phase as a result of the complex-valued scattering matrix $\hat{\theta}$. Hence, they do not cancel completely but leave a residue proportional to $\hat{\theta}-\hat{\theta}^{*}$.

The arrival time of this residue has no physical meaning, but its amplitude has. According to equation 29, it is proportional to the energy scattered by the point scatterer (Carney et al., 2004; Vasconcelos et al., 2009; Curtis and Halliday, 2010). By adding the fourth term of equation $10, \mathcal{L}\left(G_{A}^{s}, G_{B}^{s}\right)$, resulting from the crosscorrelation of the scattered Green's functions, the residual event at $t_{B}-t_{A}$ $=0.033 \mathrm{~s}$ is canceled, as shown in Figure 12 .

\section{DISCUSSION}

Our theory and examples clearly show that seismic interferometry applied to scattered wavefields cannot be explained with the first-or-

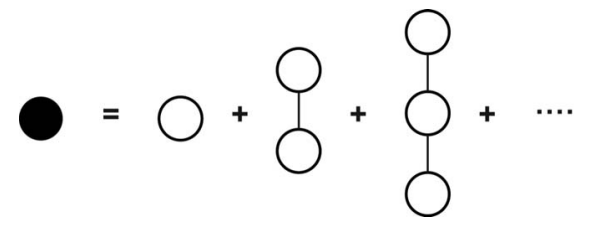

Figure 10. Diagram of the nonlinear scattering matrix (after Snieder, 1999). The single line denotes the regularized return Green's function $\hat{\bar{G}}^{\text {reg }}(\mathbf{0}, \mathbf{0}, \omega)$. The open circle denotes the linearized scattering matrix $\hat{\theta}_{1}(\omega)$, and the black circle stands for the nonlinear scattering matrix $\hat{\theta}(\omega)$. 
der Born approximation. Even for a single point scatterer, interferometry includes the crosscorrelation of two scattered responses, which is proportional to second- (and higher) order scattering. This nonlinear term can be handled consistently only when the contributing responses contain the proper nonlinear scattering effects.

Although the example we discuss is simple, it shows the relevance of taking nonlinear scattering at point scatterers into account. This does not only apply to applications in seismic interferometry. For example, Groenenboom and Snieder (1995) analyze the transmission of waves through a distribution of point scatterers. With numerical modeling experiments, they show it is essential that not only multiple scattering between the scatterers be taken into account but also that each scatterer be treated nonlinearly. Hence, accounting for the local nonlinearity of scatterers is essential in modeling (Groenenboom and Snieder, 1995), nonlinear inversion (Weglein et al., 2003), and interferometry of responses of scattering media (van Manen et al., 2006).

Also for the analysis of seismic migration, which is usually considered a linear process, the nonlinearity of point scatterers may be relevant. The resolution properties of seismic migration for a specific acquisition configuration are often expressed in terms of the pointspread function (or spatial resolution function), which is defined as
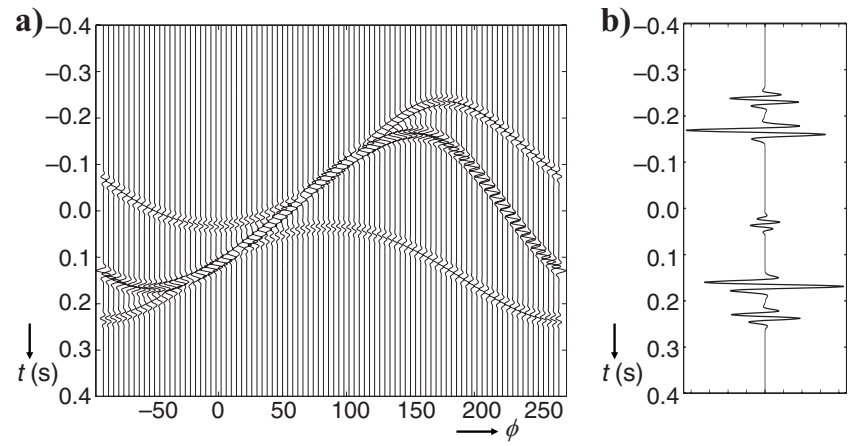

Figure 11. (a) Three-term approximation of equation 10, as in Figure $6 \mathrm{a}$, but this time for the nonlinear scatterer model of equation 30 . (b) The sum of all traces in (a). The nonphysical arrivals at $t_{B}-t_{A}$ $=0.033 \mathrm{~s}$ do not cancel but leave a residue with an amplitude proportional to the energy scattered by the point scatterer.

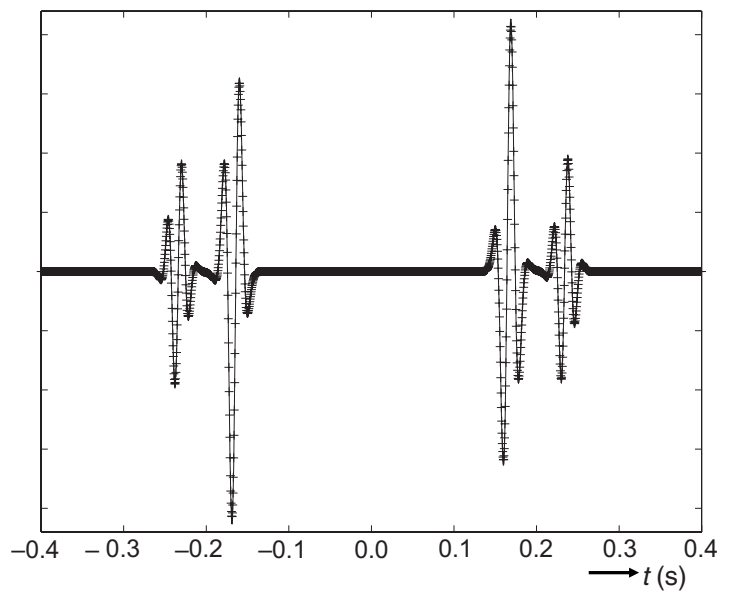

Figure 12. All four terms of equation 10, as in Figure 9, but this time for the nonlinear scatterer model of equation 30 . The fourth term cancels the residual event of Figure 11b. the migration result of the response of a single point scatterer (Miller et al., 1987; Schuster and Hu, 2000; Gelius et al., 2002; Lecomte, 2008). Moreover, the point-spread function is sometimes used in migration deconvolution to improve spatial resolution (Hu et al., 2001; Yu et al., 2006). In both approaches, the linearized point scatterer model could be replaced by the nonlinear model of equation 28 to obtain a more accurate point-spread function. A caveat for the application in migration deconvolution is that the amplitude as well as the phase of the nonlinear model depend on the local contrast parameter.

\section{CONCLUSIONS}

Starting with the Green's function representation for seismic interferometry, we have derived the generalized optical theorem and, subsequently, the nonlinear scattering matrix of a point scatterer. We have closed the loop by analyzing seismic interferometry for the response of a point scatterer. None of the theories discussed is new, but some of the derivations are new and the relations between the theories are insightful. One main conclusion is that the Born approximation is an insufficient model to explain all aspects of seismic interferometry, even for a single point scatterer. This conclusion makes sense if one bears in mind that the Born approximation does not conserve energy, whereas the interferometric Green's function representation is a generalization of the energy conservation law. Last but not least, we have indicated the relevance of the nonlinear scattering matrix of a point scatterer for modeling, inversion, and migration.

\section{ACKNOWLEDGMENTS}

This work is supported by the Netherlands Research Centre for Integrated Solid Earth Science (ISES) and by the U. S. National Science Foundation through grant EAS-0609595. We thank associate editor Ivan Vasconcelos, reviewer David Halliday, and two anonymous reviewers for their valuable comments and suggestions, which helped improve this paper.

\section{REFERENCES}

Bojarski, N. N., 1983, Generalized reaction principles and reciprocity theorems for the wave equations, and the relationship between the time-advanced and time-retarded fields: Journal of the Acoustical Society of America, 74, 281-285.

Born, M., and E. Wolf, 1965, Principles of optics: Pergamon Press.

Butkov, E., 1968, Mathematical physics: Addison-Wesley.

Carney, P. S., J. C. Schotland, and E. Wolf, 2004, Generalized optical theorem for reflection, transmission, and extinction of power for scalar fields: Physical Review E, 70, 036611-1-036611-7.

Curtis, A., and D. Halliday, 2010, Directional balancing for seismic and general wavefield interferometry: Geophysics, 75, no. 1, SA1-SA14.

de Hoop, A. T., 1988, Time-domain reciprocity theorems for acoustic wave fields in fluids with relaxation: Journal of the Acoustical Society of America, 84, 1877-1882.

Fokkema, J. T., and P. M. van den Berg, 1993, Seismic applications of acoustic reciprocity: Elsevier Scientific Publ. Co., Inc.

Gelius, L.-J., I. Lecomte, and H. Tabti, 2002, Analysis of the resolution function in seismic prestack depth imaging: Geophysical Prospecting, 50, 505-515.

Glauber, R., and V. Schomaker, 1953, The theory of electron diffraction: Physical Review, 89, 667-671.

Groenenboom, J., and R. Snieder, 1995, Attenuation, dispersion, and anisotropy by multiple scattering of transmitted waves through distributions of scatterers: Journal of the Acoustical Society of America, 98, 3482-3492.

Halliday, D., and A. Curtis, 2009a, Generalized optical theorem for surface waves and layered media: Physical Review E, 79, 056603.

, 2009 b, Seismic interferometry of scattered surface waves in attenuative media: Geophysical Journal International, 178, 419-446.

Heisenberg, W., 1943, Die "beobachtbaren Größen" in der Theorie der El- 
ementarteilchen: Zeitschrift für Physik, 120, 513-538.

Hu, J., G. T. Schuster, and P. Valasek, 2001, Poststack migration deconvolution: Geophysics, 66, 939-952.

Larose, E., L. Margerin, A. Derode, B. van Tiggelen, M. Campillo, N. Shapiro, A. Paul, L. Stehly, and M. Tanter, 2006, Correlation of random wavefields: An interdisciplinary review: Geophysics, 71, no. 4, SI11-SI21.

Lecomte, I., 2008, Resolution and illumination analyses in PSDM: A raybased approach: The Leading Edge, 27, 650-663.

Marston, P. L., 2001, Generalized optical theorem for scatterers having inversion symmetry: Applications to acoustic backscattering: Journal of the Acoustical Society of America, 109, 1291-1295.

Miller, D., M. Oristaglio, and G. Beylkin, 1987, A new slant on seismic imaging: Migration and integral geometry: Geophysics, 52, 943-964.

Morse, P. M., and H. Feshbach, 1953, Methods of theoretical physics I: McGraw-Hill Book Company, Inc.

Newton, R. G., 1976, Optical theorem and beyond: American Journal of Physics, 44, no. 7, 639-642.

Oristaglio, M. L., 1989, An inverse scattering formula that uses all the data: Inverse Problems, 5, 1097-1105.

Porter, R. P., 1970, Diffraction-limited, scalar image formation with holograms of arbitrary shape: Journal of the Optical Society of America, 60 1051-1059.

Rodberg, L. S., and R. M. Thaler, 1967, Introduction to the quantum theory of scattering: Academic Press, Inc.

Schuster, G. T., 2009, Seismic interferometry: Cambridge University Press.

Schuster, G. T., and J. Hu, 2000, Green's function for migration: Continuous recording geometry: Geophysics, $\mathbf{6 5}, 167-175$.

Snieder, R., 1999, Imaging and averaging in complex media, in J. P. Fouque, ed., Diffuse waves in complex media: Kluwer Academic Publ., 405-454.

Snieder, R., M. Miyazawa, E. Slob, I. Vasconcelos, and K. Wapenaar, 2009a, A comparison of strategies for seismic interferometry: Surveys in Geophysics, 30, 503-523.
Snieder, R., F. J. Sánchez-Sesma, and K. Wapenaar, 2009b, Field fluctuations, imaging with backscattered waves, a generalized energy theorem, and the optical theorem: SIAM Journal on Imaging Sciences, 2, 763-776.

Snieder, R., K. van Wijk, M. Haney, and R. Calvert, 2008, Cancellation of spurious arrivals in Green's function extraction and the generalized optical theorem: Physical Review E, 78, 036606.

Thorbecke, J., and K. Wapenaar, 2007, On the relation between seismic interferometry and the migration resolution function: Geophysics, 72, no. 6 , T61-T66.

van Manen, D.-J., A. Curtis, and J. O. A. Robertsson, 2006, Interferometric modeling of wave propagation in inhomogeneous elastic media using time reversal and reciprocity: Geophysics, 71, no. 4, SI41-SI60.

van Manen, D.-J., J. O. A. Robertsson, and A. Curtis, 2005, Modeling of wave propagation in inhomogeneous media: Physical Review Letters, 94 164301-1-164301-4.

van Rossum, M. C. W., and Th. M. Nieuwenhuizen, 1999, Multiple scattering of classical waves: Microscopy, mesoscopy, and diffusion: Reviews of Modern Physics, 71, 313-371.

Vasconcelos, I., R. Snieder, and H. Douma, 2009, Representation theorems and Green's function retrieval for scattering in acoustic media: Physica Review E, 80, 036605.

Wapenaar, K., and J. Fokkema, 2006, Green's function representations for seismic interferometry: Geophysics, 71, no. 4, SI33-SI46.

Wapenaar, K., J. Fokkema, and R. Snieder, 2005, Retrieving the Green's function in an open system by cross-correlation: A comparison of approaches (L): Journal of the Acoustical Society of America, 118, 2783-2786.

Weglein, A. B., F. V. Araújo, P. M. Carvalho, R. H. Stolt, K. H. Matson, R. T. Coates, D. Corrigan, D. J. Foster, S. A. Shaw, and H. Zhang, 2003, Inverse scattering series and seismic exploration: Inverse Problems, 19, R27-R83.

Yu, J., J. Hu, G. T. Schuster, and R. Estill, 2006, Prestack migration deconvolution: Geophysics, 71, no. 2, S53-S62. 